\title{
Joint User and Relay Selection based Cooperative NOMA with Imperfect CSI
}

\author{
ARADHANA MISRA, KANDARPA KUMAR SARMA, NIKOS E. MASTORAKIS ${ }^{3}$ \\ ${ }^{1,2}$ Department of Electronics and Communication Engineering, \\ ${ }^{1,2}$ Gauhati University, Guwahati, Assam, India-781022 \\ ${ }^{3}$ Technical University of Sofia, Sofia, Bulgaria \\ ${ }^{1,2}$ INDIA, ${ }^{3}$ BULGARIA
}

aradhana.misra@gauhati.ac.in,kandarpaks@gauhati.ac.in,mastor@tu-sofia.bg

\begin{abstract}
In this paper we consider the deployment of mobile relay nodes in a Cooperative Non Orthogonal Multiple Access (CNOMA) system with variable gain Amplify and Forward relaying. In a multiuser downlink scenario, two users are chosen as the NOMA destination pairs based on the channel quality and another suitable user is chosen to act as the relay node. Two standard relay selection protocols are examined here viz. the partial relay selection (PRS) and optimal relay selection (ORS). An outdated channel condition induced by the user mobility condition is considered in this paper and the impact of channel correlation on the system performance is analyzed. The outage performance and ergodic sum rate capacity of the users is observed under the two relay selection schemes. Further, the impact of user node velocity on the relay selection and outage performance have been reported. It is observed that the optimal relay selection has an overall better performance compared to partial relay selection. However, the impact of user velocity and time delay is lesser in case of partial relay selection. Hence, partial relay selection method has a more stable performance in case of imperfect CSI compared to optimal relay selection.
\end{abstract}

Key-Words: - NOMA, Cooperative Relaying, Partial Relay Selection, Optimal Relay Selection, Imperfect CSI Received: June 1, 2019. Revised: December 26, 2019. Accepted: January 2, 2020. Published: February 5, 2020.

\section{Introduction}

Non Orthogonal Multiple Access (NOMA), incorporated in the Fifth generation (5G) of wireless standards, is a spectrally efficient technique that can meet the massive data requirements by exploiting multiple user transmission in power domain. The main technique of NOMA is to use superposition coding in the transmitter side to combine multiple signals and to use successive interference cancellation (SIC) at the receiver side to detect the signals [1]. Multiple access is achieved in the downlink scenario by allocating less power to the users with comparatively stronger channels and more power to the weaker channel users. NOMA can be extended to a cooperative network to enhance the transmission reliability of users with poor channel conditions, [2]. Cooperative NOMA with dedicated relaying is an important paradigm of wireless standards that can increase the spatial diversity and improve overall system performance such as bit error rate, outage probability and data throughput. The outage performance of two relaying protocols, the Decode and Forward (DF) and Amplify and Forward (AF) relaying in a NOMA network has been widely analyzed in a dual hop architecture in various literatures [3]-[6]. While DF relaying decodes the information at the relay node, and then retransmits it after encoding again, in AF protocol, the received 
signal is first scaled up by a gain factor and then retransmitted to the destination [6].

In a multi-user, multi-relay cooperative network, user selection and relay selection schemes are applied to choose the best possible set of user and relay nodes for cooperative communication at a certain instant of time [7]. Not all users and relays are deemed capable of cooperation and the choice of a suitable set is dependent on the availability of Channel State Information (CSI), reduction of signaling overhead and complexity of the selection algorithm. Partial relay selection and optimal relay selection are two popular selection strategies that has been adopted in dual hop architecture in various literature [8], [9]. While PRS scheme is based on CSI of either the source to relay (S-R) or relay to destination (R-D) link, the ORS scheme considers CSI of both links to make the best choice of the candidate relay. Thus, in optimal relay selection method, the better outage performance is traded off with increased signaling overhead for CSI feedback. The outage performance and ergodic sum rate of two stage relay selection has been studied in [4], which guarantees the best performance of one user while considering acceptable performance of the other NOMA user. Similar work on closed form expression of outage performance has been carried out in multi-hop environment with immediate best path selection and parallel best path selection in [10].

Relay selection in a cooperative NOMA network in [2]-[10] considers perfect CSI availability at the communication nodes. However, it is not practical to assume perfect CSI for both relay and user selection as well as for decoding the signal at the receiver. The CSI often becomes outdated because of feedback delay as well as longer updating time and also contains estimation errors because of noise. As such a relay selected at a particular time may not be optimal for transmission of information because of the imperfect CSI and hence result in degradation in the system performance. Closed form expression for outage probability of each user in an energy harvested cooperative NOMA network is considered in [11] and a max-min relay selection scheme in the same set up is reported in [12]. In [13], a cooperative NOMA with user relay which is selected from among the neighboring relays is analyzed in presence of imperfect CSI. A decode and forward Cooperative Cognitive Radio (CR) NOMA network is reported in [14]. Specifically AF relaying in Imperfect CSI is studied in [15] and [16] and with partial relay selection in [17]. The impact of imperfect CSi in an energy harvesting cooperative network with fixed and dynamic power allocation is studied in [18]. A D2D communication with cooperative NOMA and two stage power allocation is analyzed in [19] while impact of uplink transmission in a cooperative NOMA relay network is reported in [20].

While the aforementioned literature considers fixed relay nodes to achieve spatial diversity, the possibility of a hybrid architecture with user nodes taking part in cooperation has been studied in [21]. User relaying selects a probable relay node from among the available users which are not participating in the NOMA transmission at the current instant. A user with a strong channel condition can act as relay to decode and retransmit the information of another weak user. Outage performance of user relaying with DF relaying protocol has been considered in [6]. The use of a user node as relay provides advantage of lower hardware implementation cost and can exploit the possibility of better link conditions than fixed relays. Further, the choice of paired users or user pairing to share the downlink channel, is a problem of particular concern in a NOMA system. The paired users should be in good channel state so as to increase the sum rate and be easily distinguishable at the receiver end [22].

While research on relay selection and cooperative NOMA with Imperfect CSI has progressed to a good extent, different from the other available literature we consider AF relaying and the mobility condition of user and relay nodes to observe its impact on the outage performance. Further, the system model in this paper considers a direct link between the source and destination in addition to the relay link, to improve the overall system performance. The main contributions of this are as follows:

1. We consider user and relay selection in a multiuser, cooperative AF relay NOMA network with imperfect CSI.

2. We analyze the outage performance of two relay selection schemes: partial relay selection and optimal relay selection in the mentioned relay framework.

3. We consider the user and relay nodes to be mobile and observe the impact of mobility on the outage performance. 
The rest of the paper is organized as follows. In Section II, we introduce the system model and the channel model along with the joint user and relay selection schemes. In Section III, we present the outage probability analysis and system throughput. The results obtained through Monte Carlo simulations are discussed in Section IV. A conclusion to the work is provided in Section V.

\section{System Model}

In this paper we consider a cooperative NOMA network which consists of a source $\mathrm{S}$, and a set $\mathscr{M}=\left\{\mathrm{U}_{\mathrm{i}} \mid \mathrm{i}=1,2 \ldots \mathrm{M}\right\}$, of $\mathrm{M}$ users uniformly distributed around the source, from which two users (considered as destinations $\mathrm{D}_{1}$ and $\mathrm{D}_{2}$ ) are served by the source at a time and information is relayed to the destinations by one of the suitable users (considered as relay node $\mathrm{R}$ ) in the set. There exists a direct link $\left(\mathrm{S} \rightarrow \mathrm{D}_{\mathrm{k}}\right)$ and a relay link $\left(\mathrm{S} \rightarrow \mathrm{R} \rightarrow \mathrm{D}_{\mathrm{k}}\right)$, where $\mathrm{k}=\{1,2\}$ for transfer of information from the source to the destination. We consider AF relaying where the received signal at relay is amplified with gain $G$ and is retransmitted to the destinations. Each node in the network is assumed to be equipped with a single antenna and operate in a half-duplex mode. Without loss of generality, we assume that the wireless channels of all $\mathrm{S} \rightarrow \mathrm{U}_{\mathrm{i}}$ links and $\mathrm{R} \rightarrow \mathrm{D}_{\mathrm{k}}$ links are independent and identical with Rayleigh fading. Let $h_{S D k}, h_{S R}$ and $h_{R D k}$ denote the channel coefficients between the $\mathrm{S} \rightarrow \mathrm{D}_{\mathrm{k}}, \mathrm{S} \rightarrow \mathrm{R}$ and $\mathrm{R} \rightarrow \mathrm{D}_{\mathrm{k}}$ links respectively. The channel coefficients between nodes $m$ and $n$, such that $m \in\{S, R\}$ and $n \in\left\{D_{k}, R\right\}$ are modelled as circular symmetric Complex Gaussian random variables i.e. $h_{m n} \sim \mathcal{C N}\left(0, \lambda_{m n}\right)$. The corresponding channel gain $\left|h_{m n}\right|^{2}$ is exponentially distributed with parameter $1 / \lambda_{m n}$.

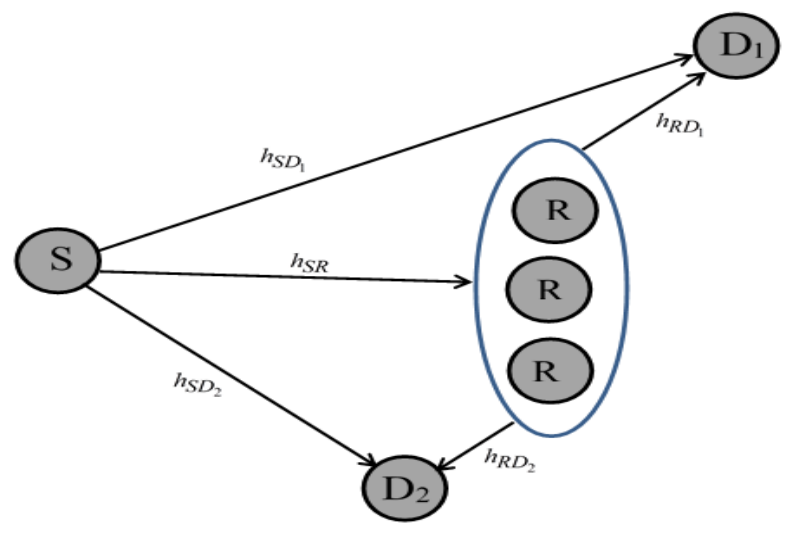

Fig.1 System model of Cooperative NOMA
The pdf of the channel gain $\left|h_{m n}\right|^{2}$ is given by

$$
f_{\left|h_{m n}\right|^{2}}(x)=\frac{1}{\lambda_{m n}} e^{-\frac{x}{\lambda_{m n}}}
$$

We also assume that zero mean and unit variance Additive White Gaussian Noise (AWGN) is present in each of the nodes. Hence the average SNR of the $\mathrm{S} \rightarrow \mathrm{D}_{\mathrm{k}}$ link is $\bar{\gamma}_{S D_{k}}=E\left\{\left|h_{S D k}\right|^{2}\right\}=\lambda_{S D k}$. Similarly, the average $\mathrm{SNR}$ of the $\mathrm{S} \rightarrow \mathrm{R}$ and $\mathrm{R} \rightarrow \mathrm{D}_{\mathrm{k}}$ links can be given by $\bar{\gamma}_{S R}$ and $\bar{\gamma}_{R D_{k}}$ respectively.

The prime focus of this paper is to investigate the effect of user mobility on the relay selection and its outage performance. The channel coefficients might change due to feedback delay inherent in the network and Doppler shift caused by the mobility of the nodes. Hence the relay selected at time $t$ may not offer the best relaying path at time $t+\tau$ because the CSI becomes outdated and hence the system performance degrades. The relationship between the channel coefficient $h_{m n}$ during relay selection and channel coefficient $\hat{h}_{m n}$ during information transfer can be modelled according to Markov's chain model for channel state evolution and it is given as

$$
\hat{h}_{m n}=\rho h_{m n}+\sqrt{1-\rho^{2}} \varepsilon_{m n}
$$

Where, $0<\rho<1$. $\rho$ is the correlation between the channel coefficients $\hat{h}_{m n}$ and $h_{m n}$ and represents the fluctuation rate of the channel, given by $\rho=$ $J_{o}\left(2 \pi \tau f_{D}\right)$, where $\mathrm{J}_{\mathrm{o}}$ represents the Zero-th order Bessel function of First kind, $f_{D}$ is the Doppler shift and $\tau$ is the time delay. Like $h_{m n}, \hat{h}_{m n}$ is also modelled as circular symmetric Complex Gaussian random variable i.e. $\hat{h}_{m n} \sim \mathcal{C N}\left(0, \lambda_{m n}\right) . \quad \varepsilon_{m n}$ is the channel error due to temporal changes in the channel and is a circular symmetric Complex Gaussian random variable i.e. $\varepsilon_{m n} \sim \mathcal{C N}(0,1)$.

According to NOMA principle, served users should have strongly distinguishable channel conditions and hence we define a User Selection criteria to select the best suitable destination pairs. Further we consider two standard relay selection (RS) schemes, for obtaining the best relaying path in the network. The User and Relay Selection schemes are discussed in the following sub sections.

\subsection{User Selection}

This work considers NOMA user pairing through a user selection process in the set of available users in 
the network. The two users with the weakest and the strongest channel coefficients are chosen as the destinations $\mathrm{D}_{1}$ and $\mathrm{D}_{2}$ respectively. The user selection criteria based on link condition can therefore be stated as

$$
\begin{aligned}
& D_{1}: \quad f^{*}=\arg \min \left(\left|h_{S U_{i}}\right|^{2}\right) ; \quad i \in\{1 \ldots M\} \\
& D_{2}: \quad n^{*}=\arg \max \left(\left|h_{S U_{i}}\right|^{2}\right) ; \quad i \in\{1 \ldots M\}
\end{aligned}
$$

where $f^{*}$ and $n^{*}$ are the selected user indices. Therefore users $U_{f^{*}}$ and $U_{n^{*}}$ are chosen as the destinations $D_{1}$ and $D_{2}$ respectively.

\subsection{Partial Relay Selection}

The partial relay selection criteria is based on the link condition and the spare user (not involved in data transmission) that has the strongest link with the BS is chosen as the relay node to forward information to the destination nodes. The selected relay index is given by Eq. (5)

$$
r^{*}=\arg \max \left(\left|h_{S U_{i}}\right|^{2}\right) ;
$$

such that $U_{i} \in \mathcal{M}$ and $U_{i} \neq U_{f^{*}}, U_{i} \neq U_{n^{*}}$.

Therefore, user $U_{r} *$ is chosen to act as the relay node R.

\subsection{Optimal Relay Selection}

The optimal relay selection criteria considers CSI availability of both the source to relay as well as relay to destination links. The relay index obtained by optimal relay selection may be written as in Eq. (6),

$$
r^{*}=\arg \max \left\{\min \left(\left|h_{S U_{i}}\right|^{2},\left|h_{U_{i} U_{f^{*}}}\right|^{2}\right\}\right.
$$

such that $U_{i} \in \mathcal{M}$ and $U_{i} \neq U_{f^{*}}, U_{i} \neq U_{n^{*}}$.

Since, this method of relay selection uses more link information, it definitely leads to the choice of best relay. But at the same time, there is increased requirement of signaling overhead which may lead to spectrum inefficiency and higher complexity.

Considering the constraints of half duplex transmissions, data transmission is assumed to take place in two time slots.

During the first time slot, the BS broadcasts the superimposed information of $\mathrm{D}_{1}$ and $\mathrm{D}_{2}$ to all the users and in the second time slot, the selected user relay amplifies and forwards the information to the destination pair. At the destinations $\left(\mathrm{D}_{1}\right.$ and $\left.\mathrm{D}_{2}\right)$, both signals from the BS (direct link) as well as the relay (indirect link) are combined together using Selection Combining (SC) during the second time slot.

The BS transmits the superimposed signal $x_{S}$ as in Eq. (7) to the relay and destinations in the first time slot,

$$
x_{s}=\sqrt{a_{1} P_{S}} x_{1}+\sqrt{a_{2} P_{S}} x_{2}
$$

where, $P_{S}$ is the transmit power of the source, $x_{1}$ and $x_{2}$ are the information signals for $\mathrm{D}_{1}$ and $\mathrm{D}_{2}$ respectively and $a_{1}$ and $a_{2}$ are the corresponding power allocation coefficients. Since according to the user selection criteria $\left|h_{S D_{1}}\right|^{2}<\left|h_{S D_{2}}\right|^{2}$, we assume that $\left|a_{1}\right|>\left|a_{2}\right|$ and $a_{1}+$ $a_{2}=1$. Fixed power allocation is assumed throughout the paper.

The received signals at the relay $\mathrm{R}$ and the destinations $D_{1}$ and $D_{2}$ are given by Eq. (8)Eq.(10)

$$
\begin{aligned}
& y_{S R}=\hat{h}_{S R} x_{s}+n_{S R} \\
& y_{S D 1}=\hat{h}_{S D 1} x_{s}+n_{S D 1} \\
& y_{S D 2}=\hat{h}_{S D 2} x_{s}+n_{S D 2}
\end{aligned}
$$

where $n_{S R}, n_{S D 1}$ and $n_{S D 2}$ are the AWGN present at relay $\mathrm{R}$ and destinations nodes $\mathrm{D}_{1}$ and $D_{2}$ respectively.

During the second time slot, the relay retransmits the signal $y_{S R}$ received during first time slot, after amplification by the relay gain $G$. The amplification factor $\mathrm{G}$ is dependent on the CSI of the source to relay link at the signal transmission time and is given by Eq. (11) as

$$
G=\sqrt{\frac{P_{R}}{P_{S}\left|\widehat{h}_{S R}\right|^{2}+N_{0}}}
$$

where $N_{0}$ is the noise power spectral density of the AWGN at the relay node. The transmitted signal is therefore given by Eq. (12).

$$
x_{R}=G y_{S R}
$$

The received signals at $\mathrm{D}_{1}$ and $\mathrm{D}_{2}$ during the second time slot are given by Eq. (13)-Eq.(14). 
$y_{R D 1}=G \hat{h}_{R D 1} \hat{h}_{S R} x_{S}+G \hat{h}_{R D 1} n_{S R}+n_{R D 1}$

$y_{R D 2}=G \hat{h}_{R D 2} \hat{h}_{S R} x_{S}+G \hat{h}_{R D 2} n_{S R}+n_{R D 2}$

We now calculate the received signal to interference and noise ratio (SINR) at $\mathrm{D}_{1}$ and $\mathrm{D}_{2}$ during the two time slots. During the first time slot, $\mathrm{D}_{1}$ decodes its own information $x_{1}$ treating $x_{2}$ as interference, with SINR

$$
\gamma_{S D 1}=\frac{a_{1} \rho_{S}\left|\widehat{h}_{S D 1}\right|^{2}}{a_{2} \rho_{S}\left|\widehat{h}_{S D 1}\right|^{2}+1}
$$

SIC is performed at the receiver $\mathrm{D}_{2}$ to detect $x_{1}$ with the following SINR,

$$
\gamma_{S D 2}^{1}=\frac{a_{1} \rho_{S}\left|\widehat{h}_{S D 2}\right|^{2}}{a_{2} \rho_{S}\left|\widehat{h}_{S D 2}\right|^{2}+1}
$$

After subtraction of $x_{1}$ from $y_{S D 2}$, the signal $x_{2}$ is decoded with an SINR,

$$
\gamma_{S D 2}^{2}=a_{2} \rho_{S}\left|\hat{h}_{S D 2}\right|^{2}
$$

During the cooperative phase, $\mathrm{D}_{1}$ detects $x_{1}$ with the following SINR,

$$
\gamma_{R D 1}=\frac{G^{2}\left|\widehat{h}_{R D 1}\right|^{2}\left|\widehat{h}_{S R}\right|^{2} a_{1} \rho_{S}}{G^{2}\left|\widehat{h}_{R D 1}\right|^{2}\left|\widehat{h}_{S R}\right|^{2} a_{2} \rho_{S}+G^{2}\left|\widehat{h}_{R D 1}\right|^{2}+1}
$$

At $\mathrm{D}_{2}, x_{1}$ is detected treating $x_{2}$ as an interference. SINR at $\mathrm{D}_{2}$ to detect $x_{1}$ is given by

$$
\gamma_{R D 2}^{1}=\frac{G^{2}\left|\widehat{h}_{R D 2}\right|^{2}\left|\widehat{h}_{S R}\right|^{2} a_{1} \rho_{S}}{G^{2}\left|\widehat{h}_{R D 2}\right|^{2}\left|\widehat{h}_{S R}\right|^{2} a_{2} \rho_{S}+G^{2}\left|\widehat{h}_{R D 2}\right|^{2}+1}
$$

After detecting and subtracting $x_{1}, x_{2}$ is decoded with an SINR

$$
\gamma_{R D 2}^{2}=\frac{G^{2}\left|\widehat{h}_{R D 2}\right|^{2}\left|\widehat{h}_{S R}\right|^{2} a_{2} \rho_{S}}{G^{2}\left|\widehat{h}_{R D 2}\right|^{2}+1}
$$

Since selection combining is used at the receiver, the best signal received during the two time slots is considered and hence the instantaneous SINRs at $\mathrm{D}_{1}$ and $\mathrm{D}_{2}$ over the transmission time are given by Eq. (21) and Eq. (22) respectively, as

$$
\begin{array}{r}
\gamma_{D 1}=\max \left(\gamma_{S D 1}, \gamma_{R D 1}\right) \\
\gamma_{D 2}=\max \left(\gamma_{S D 2}^{2}, \gamma_{R D 2}^{2}\right)
\end{array}
$$

An outage event at $D_{1}$ is said to have occurred if the SINR at $D_{1}$ falls below threshold SINR during direct as well as cooperative phase. Therefore, outage probability of $\mathrm{D}_{1}$ is given by

$$
\begin{aligned}
\mathcal{O}_{D 1} & =\operatorname{Pr}\left(\gamma_{S D 1}<\gamma_{t h 1}\right) \operatorname{Pr}\left(\gamma_{R D 1}<\gamma_{t h 1}\right) \\
& =\operatorname{Pr}\left(\max \left(\gamma_{S D 1}, \gamma_{R D 1}\right)<\gamma_{t h 1}\right) \\
& =\operatorname{Pr}\left(\gamma_{D 1}<\gamma_{t h 1}\right)
\end{aligned}
$$

If the target rate of $\mathrm{D}_{1}$ is $R_{1}$, then the threshold SINR for $\mathrm{D}_{1}$ to detect $x_{1}$, is defined as $\gamma_{t h 1}=$ $2^{2 R_{1}}-1$.

On the other hand, an outage event at $\mathrm{D}_{2}$ can be avoided if $\mathrm{D}_{2}$ can detect the information $x_{1}$ and $x_{2}$ in both time slots. Therefore outage probability of $\mathrm{D}_{2}$ can be stated as

$$
\begin{aligned}
& \mathcal{O}_{D 2}=\left[1-\operatorname{Pr}\left(\gamma_{S D 2}^{1}>\gamma_{t h 1}, \gamma_{S D 2}^{2}>\gamma_{t h 2}\right)\right] \\
& \times\left[1-\operatorname{Pr}\left(\gamma_{S D 2}^{1}>\gamma_{t h 1}, \gamma_{S D 2}^{2}>\gamma_{t h 2}\right)\right]
\end{aligned}
$$

where $\gamma_{t h 2}=2^{2 R_{2}}-1$ is the threshold SINR of $\mathrm{D}_{2}$ and $R_{2}$ is the corresponding rate of $\mathrm{D}_{2}$.

In this paper, we define an overall outage probability as the probability that either of the destinations is in outage condition i.e.

$$
\mathcal{O}_{\text {outage }}=\mathcal{O}_{D 1}+\mathcal{O}_{D 2}
$$

The ergodic capacities of the users $\mathrm{D}_{1}$ and $\mathrm{D}_{2}$ are given by Eq. (26) and Eq. (27).

$$
\begin{aligned}
& C_{1}=\frac{1}{2} \log _{2}\left(1+\gamma_{D 1}\right) \\
& C_{2}=\frac{1}{2} \log _{2}\left(1+\gamma_{D 2}\right)
\end{aligned}
$$

And the sum rate of the system is given as

$$
\mathrm{C}=\mathrm{C}_{1}+\mathrm{C}_{2}
$$

The performance analysis of the system considered in this paper is carried out taking system outage, the sum rate and the ergodic capacities as the performance performance metrics. 


\section{Experimental Results and Analysis}

The performance analysis of partial and optimal relay selection in a mobile relay environment is presented in this section considering imperfect CSI at the source and relay nodes. While we analyse the effect of imperfect CSI considering the correlation coefficient, we have compared the results with that of perfect CSI $(\rho=1)$ which we assume to represent a static relay with static channel conditions.

In this work, we consider a 10 user scenario and the relay is selected from among the remaining 8 users which are not being served currently by the BS. Consideration of a denser user deployment scenario effects the relay selection time and complexity without having any adverse effect on the system performance. Hence, the user density has been kept constant in all simulations and the dependence of system performance on the user density is considered to be out of scope of this paper. We observe the system performance under two different target data rates i.e. $0.5 \mathrm{bits} / \mathrm{sec} / \mathrm{Hz}$ and $1 \mathrm{bit} / \mathrm{sec} / \mathrm{Hz}$ and also assume that $\mathrm{R}_{1}=\mathrm{R}_{2}$. Some of the simulation parameters used in this work are summarized in Table 1.

Table 1 Simulation Parameters and Specifications

\begin{tabular}{|c|l|l|}
\hline $\begin{array}{c}\text { Sl. } \\
\text { No } \\
.\end{array}$ & \multicolumn{1}{|c|}{ Parameter } & Specification \\
\hline 1. & Carrier Frequency & $2.4 \mathrm{GHz}$ \\
\hline 2. & Avg. No of Users & 10 \\
\hline 3. & Target Data Rates & $\mathrm{R}_{1}=\mathrm{R}_{2}=0.5 \mathrm{bits} / \mathrm{s} / \mathrm{Hz}$ \\
\hline 4. & $\begin{array}{c}\text { Power allocation } \\
\text { coefficient }\end{array}$ & $a_{1}=0.9, a_{2}=0.1$ \\
\hline 5. & Channel profile & Rayleigh \\
\hline 6. & Channel Estimation & Ideal \\
\hline 7. & Avg. Channel Gain, & $E\left\{\left|h_{i j}\right|^{2}\right\}=1$ \\
\hline 8. & Avg. Noise Power & \multicolumn{1}{c|}{$\sigma^{2}=1$} \\
\hline
\end{tabular}

In a fixed relay network, the position of the relay nodes are chosen such that a high degree Line of Sight (LoS) path is existent between the source and relay nodes. Further, by using highly directional antenna, a superior link quality can be guaranteed at all times between the source and relay nodes. Hence the end-to-end SNR in a fixed relay network depends only on the relay to destination link is

$$
\gamma_{e-e}=\gamma_{R D}
$$

Therefore relay selection in a cooperative network with fixed relays is mostly influenced by the channel quality of the relay to destination link. Further, because of the LOS path in the source to relay link, an improved performance is guaranteed in this scenario compared to a cooperative network where mobile relay nodes are used. This is also evident from the results obtained in Fig. 2, where the outage probability of fixed and mobile relay based cooperative OMA and NOMA network has been shown. Simulation results show that fixed relay NOMA and OMA outperforms mobile relay networks but the improvement in coding gain in both cases is not more than $5 \mathrm{~dB}$. On this basis, it can be justified that mobile relaying has an overall advantage above fixed relays as it allows user devices to be used as relay nodes with only minimal increase in transmit power to attain the performance of fixed relays. This paper therefore considers mobile relay nodes where, a suitable user present in the network is selected to relay information to other users in the network.

In Fig. 3, outage performance of partial relay selection in cooperative NOMA has been shown for two different target rates of the users i.e. $\mathrm{R}=0.5 \mathrm{bits} / \mathrm{s} / \mathrm{Hz}$ and $\mathrm{R}=1 \mathrm{bit} / \mathrm{s} / \mathrm{Hz}$. As target rate increases, the outage also increases in the network. Considering relay nodes to be mobile, we show the effect of imperfect CSI on outage performance. While $\rho=1$, represents perfect CSI, we have shown the outage performance for $\rho=0.8$ and $\rho=0.7$ and it is seen that the outage performance worsens as $\rho$ decreases. 


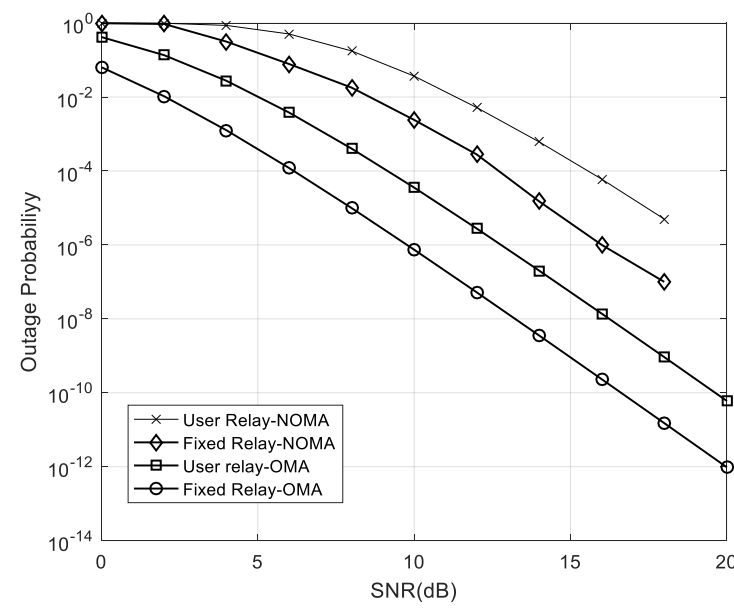

Fig.2 Outage Performance of fixed and mobile relay based OMA and NOMA network

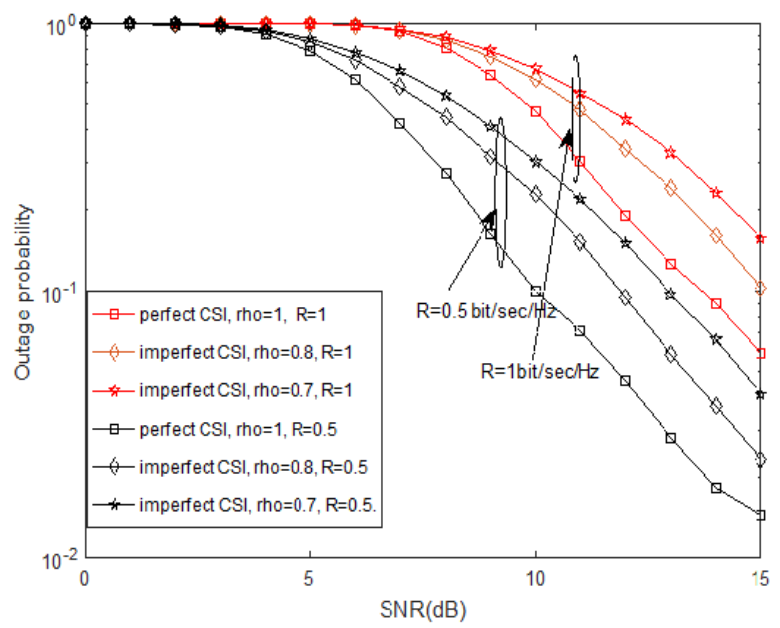

Fig.3 Outage Performance of Partial Relay Selection with Imperfect CSI $(\rho=1,0.8,0.7)$

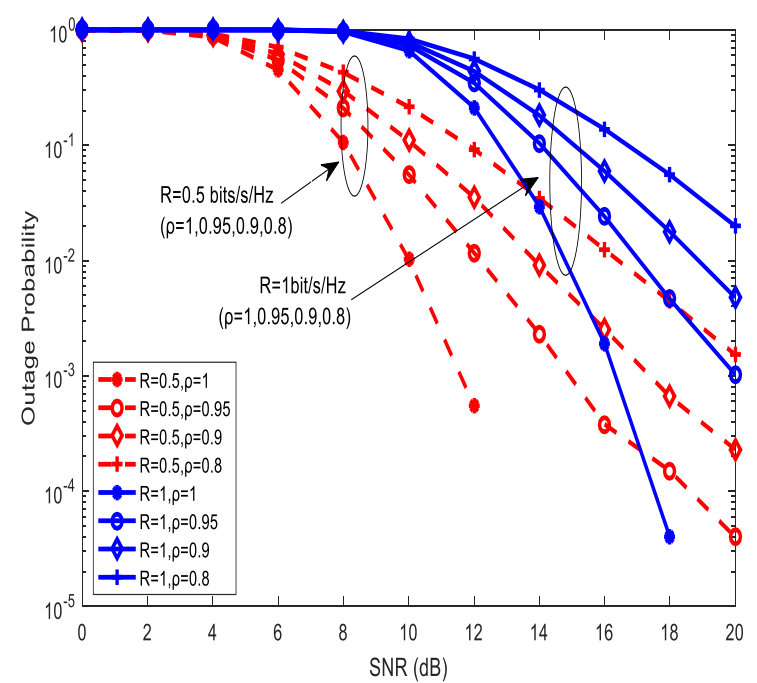

Fig 4 Outage Performance of Optimal Relay Selection with Imperfect CSI $(\rho=1,0.95,09,0.8)$
Such a situation is inevitable in a mobile relay environment where channel becomes outdated due to the Doppler shift involved and hence channel correlation has to be considered in system design and performance analysis. A similar analysis is shown in Fig.4 where we consider optimal relay selection method for two different target data rates of $\mathrm{R}=0.5 \mathrm{bits} / \mathrm{s} / \mathrm{Hz}$ and $1 \mathrm{bit} / \mathrm{s} / \mathrm{Hz}$. It is observed that for both partial as well optimal relay selection schemes, the relative impact of channel correlation is more adverse at higher SNR as compared to low SNR range.

The sum rate of the NOMA system for partial and optimal relay selections are shown in Fig. 5 and Fig. 6 respectively. The sum rate and the individual ergodic capacities analyzed for both the relay selection schemes for channel correlation values $\rho=1,0.8$ and 0.5 . We have considered the target rate to be $\mathrm{R}=1 \mathrm{bit} / \mathrm{s} / \mathrm{Hz}$ and power allocation factors of $\mathrm{a}_{1}=0.9$ and $\mathrm{a}_{2}=0.1$. In both optimal relay selection and partial relay selection, the sum rate capacity increases with increase in SNR and the rate decreases minimally as the channel correlation decreases. The improvement in outage performance is uniform throughout the SNR range. However, it is seen that for both optimal as well as partial relay selections, the effect of correlation coefficient on the outage probability is not quiet prominent and hence we can say that the sum rate is not affected by the imperfect channel conditions. From Fig. 5 and Fig. 6, it can be seen that in the low SNR regime, the ergodic capacity of weak user $\mathrm{D}_{1}$ increases with SNR but beyond SNR of $20 \mathrm{~dB}$, the rate remains essentially constant with SNR. This behavior in the rate of $\mathrm{D}_{1}$ can be observed in the case of both partial as well as optimal relay selections. The rate of $\mathrm{D}_{1}$ is not affected by the channel correlation in high SNR regime. The ergodic rate of the stronger user $\mathrm{D}_{2}$ however shows a steady increase with increase in SNR and the rates decreases as $\rho$ decreases. Using NOMA, $D_{1}$ can achieve a higher rate in the low SNR range. However, the rate of $\mathrm{D}_{2}$ i.e. the stronger user is higher in the higher SNR range, beyond $20 \mathrm{~dB}$. 


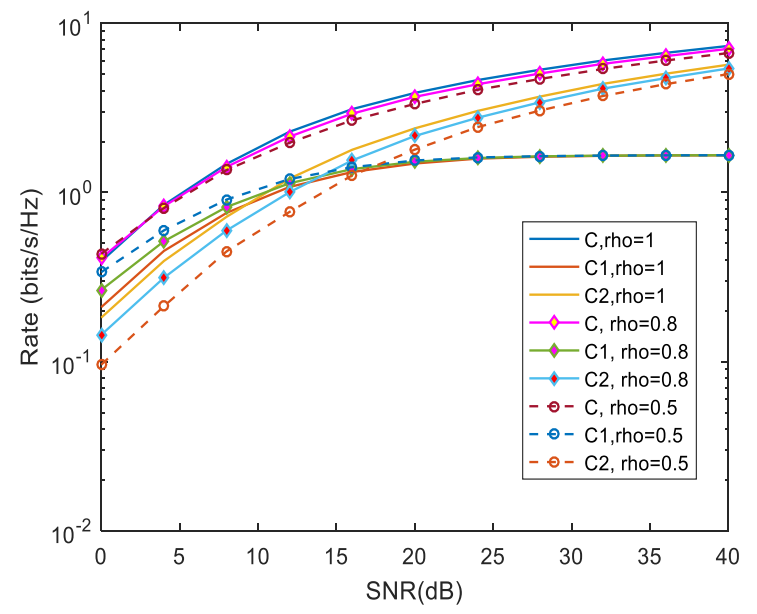

Fig. 5 Sum Rate $\left(C=C_{1}+C_{2}\right)$, Ergodic Capacities $C_{1}$ and $C_{2}$ Vs SNR for Partial Relay Selection with Imperfect CSI. $(\rho=1,0.8,0.5)$

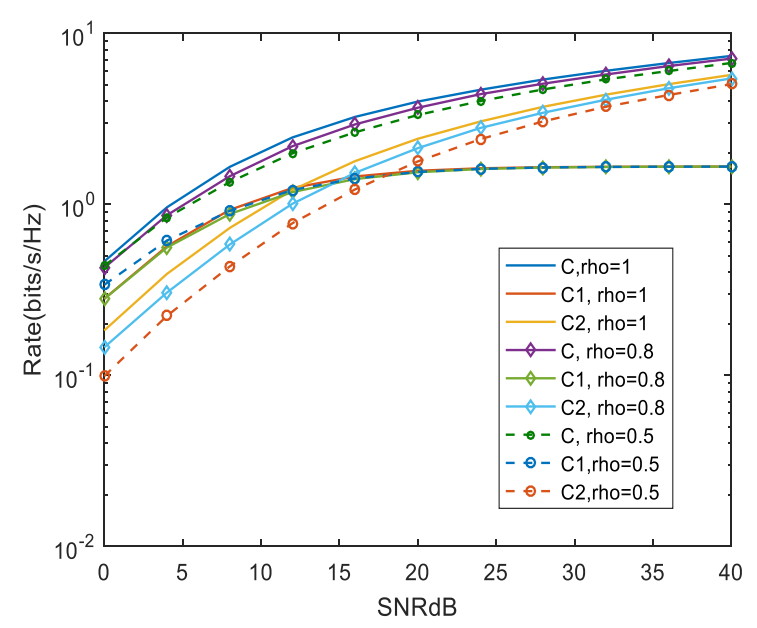

Fig. 6 Sum Rate $\left(C=C_{1}+C_{2}\right)$, Ergodic Capacities $C_{1}$ and $C_{2}$ Vs SNR for Optimal Relay Selection with Imperfect CSI. $(\rho=1,0.8,0.5)$

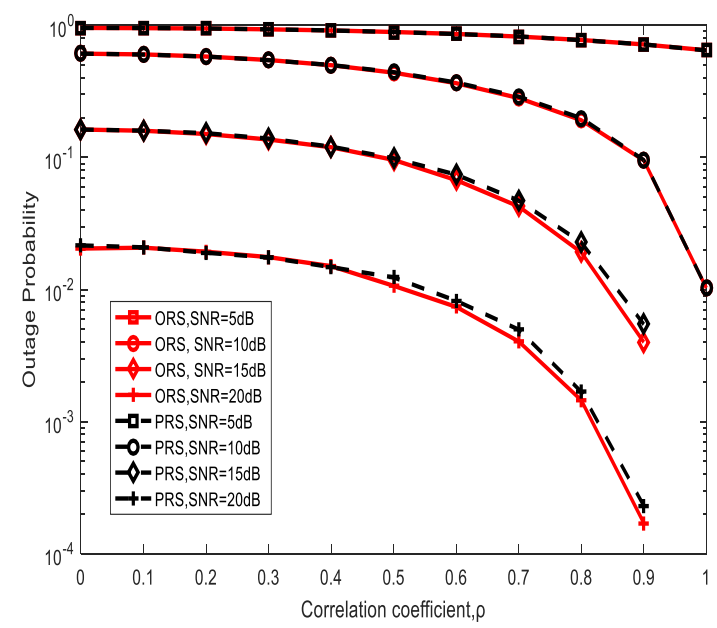

Fig. 7 Outage Probability Vs $\rho$ for Partial Relay Selection and Optimal Relay Selection $(\mathrm{R}=0.5 \mathrm{bits} / \mathrm{s} / \mathrm{Hz})$
In Fig. 7, the variation in outage probability with correlation coefficient is shown for SNR of $5,10,15$ and $20 \mathrm{~dB}$. A higher correlation coefficient signifies minimal changes in the channel between the relay selection time and information transmission time and hence the outage probability is lower in the higher range of $\rho$. Further, both optimal and relay selection techniques have near identical performance.

In Fig. 8, the outage performance of optimal relay selection is compared with variation in the relative mobile relay velocity, considering a delay of $1 \mathrm{~ms}$ and at carrier frequency of $2.4 \mathrm{GHz}$. Speed of $0 \mathrm{kmph}$ represents a static user i.e. a stationary vehicle or a fixed user. A degradation in outage performance is seen as relative velocity of the relay node increases. As velocity increases, the Doppler shift causes the channel correlation to drop and hence there is a consequent rise in the outage probability of the system. Here, we consider $\rho_{\mathrm{SR}}=\rho_{\text {RDk. }}$ i.e. we assume that the channel correlation of the $\mathrm{S} \rightarrow \mathrm{R}$ and $\mathrm{R} \rightarrow \mathrm{D}$ are same. A similar analysis is shown in Fig. 9 for the partial relay selection scheme where outage performance with different values relative relay velocity is considered with a delay of $\tau=10 \mathrm{~ms}$. Compared to the static relay case, a mobile relay has degraded outage probability and the performance worsens with increase in Doppler shift due to user relay mobility.

The effect of time delay on the outage performance is shown in Fig. 10 for optimal relay selection with relative relay velocity of 10 $\mathrm{kmph}$ and in Fig. 11 for partial relay selection with relative velocities of $10 \mathrm{kmph}$ and $40 \mathrm{kmph}$. The time delay incorporates feedback delay as well delay in transmission time from the relay selection time. Higher delay time results in a channel with lower correlation value and hence degrades the system performance. We have shown the effect of delay as low as $1 \mu$ s and upto $10 \mathrm{~ms}$ in the analysis. The system performance shows less variation for time delay lesser than $500 \mu$ s but almost represents a static channel condition with $\rho \approx 1$. With larger time delay, the 
outage performance shows considerable degradation as delay $\tau$ increases. The analysis is carried out considering a carrier frequency of 2.4 GHz.

From the analysis carried out it can be seen that, a relay node which has low ( $\approx 10 \mathrm{kmph})$ or moderate velocity $(\approx 30 \mathrm{kmph})$ can be suitably used as a relay node with acceptable degradation in outage probability provided the time delay involved is considerably low $(<500 \mu \mathrm{s})$.

\section{Conclusion}

In this paper, we have analyzed the partial and optimal relay selection schemes in a user relay cooperative NOMA system in presence of imperfect CSI. The impact of Doppler shift and time delay on the system performance has been shown. A cooperative NOMA system with mobile relay nodes has performance degradation compared to a fixed relay system. But a relay node with low to moderate velocity can be used as a suitable relay node when the time delay is very low. However, analysis performed shows that the sum rate and ergodic capacity of the system suffers less variation due to imperfect CSI.

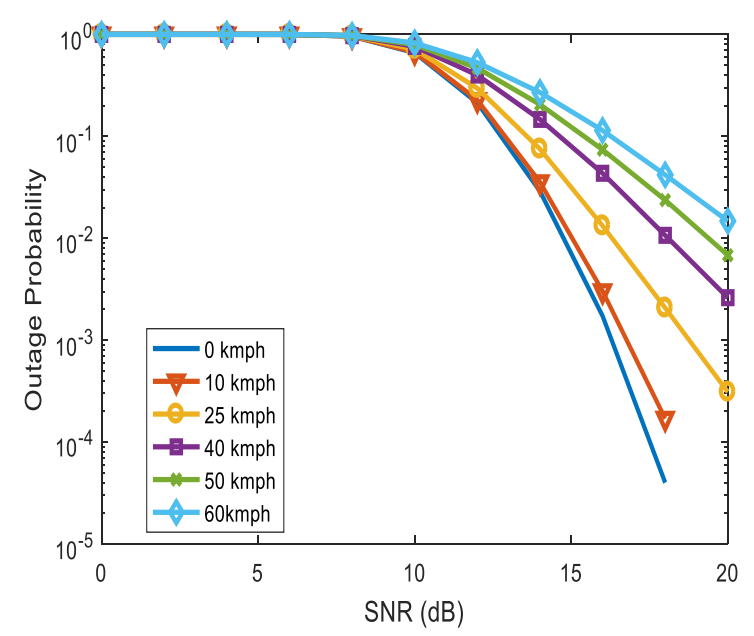

Fig 8 Outage Probability Vs SNR for Optimal Relay Selection $\left(\mathrm{R}=1 \mathrm{bits} / \mathrm{s} / \mathrm{Hz}, a_{1}=0.9 . a_{2}=0.1, \tau=1 \mathrm{~ms}, \mathrm{f}_{\mathrm{c}}=2.4 \mathrm{GHz}\right)$

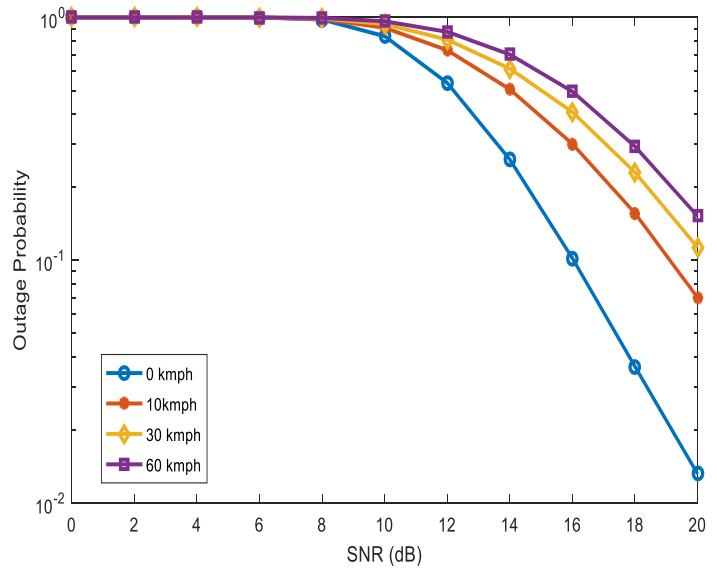

Fig. 9 Outage Probability Vs SNR for Partial Relay Selection $\left(\mathrm{R}=1 \mathrm{bits} / \mathrm{s} / \mathrm{Hz}, a_{1}=0.9 . a_{2}=0.1, \tau=10 \mathrm{~ms}, \mathrm{f}_{\mathrm{c}}=2.4 \mathrm{GHz}\right)$

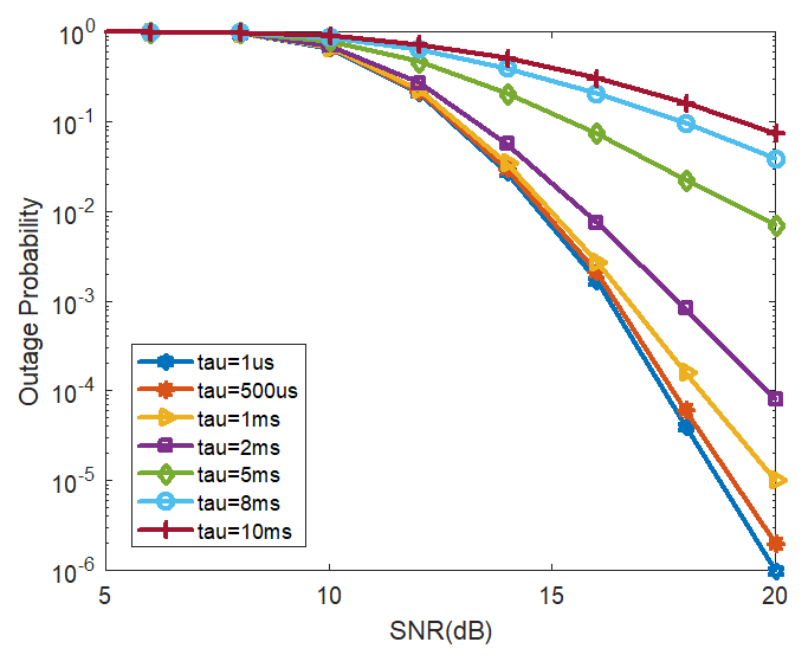

Fig. 10 Outage Probability Vs SNR for Optimal Relay Selection with varyying delay $\left(\mathrm{R}=1 \mathrm{bits} / \mathrm{s} / \mathrm{Hz}, \mathrm{v}=10 \mathrm{kmph}, \mathrm{f}_{\mathrm{c}}=2.4 \mathrm{GHz}\right)$

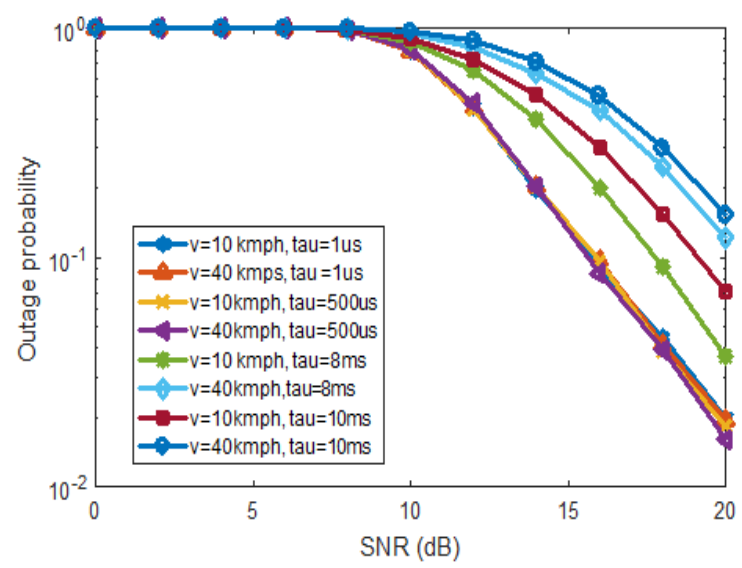

Fig. 11 Outage Probability Vs SNR for Partial Relay Selection with varying delay $\left(\mathrm{R}=1 \mathrm{bit} / \mathrm{s} / \mathrm{Hz}, \mathrm{v}=10 \mathrm{kmph}\right.$ and $40 \mathrm{kmph}, \mathrm{f}_{\mathrm{c}}=2.4$ $\mathrm{GHz}$ ) 


\section{References:}

[1] S. M. R. Islam, N. Avazov, O. A. Dobre and K. Kwak, Power-Domain Non-Orthogonal Multiple Access (NOMA) in 5G Systems: Potentials and Challenges, in IEEE Communications Surveys \& Tutorials, Vol. 19, No. 2, 2017, pp. 721-742.

[2] Z. Ding, M. Peng and H. V. Poor, Cooperative Non-Orthogonal Multiple Access in 5G systems, in IEEE Communications Letters, Vol. 19, No. 8, 2015, pp. 1462-1465.

[3] Md. B. Uddin et al., Cooperative Amplify-and Forward Relaying in NOMA", in Proceedings of Korean Institute of Communications and Information Sciences (KICS), South Korea, 2018.

[4] Q. Y. Liau and C. Y. Leow, Amplify-andForward Relay Selection in Cooperative NonOrthogonal Multiple Access, in Proceedings of IEEE 13th Malaysia International Conference on Communications (MICC), Johor Bahru, 2017 pp. $79-83$.

[5] Liang, Y. Wu, D. W. K. Ng, Y. Zuo, S. Jin and H. Zhu, Outage Performance for Cooperative NOMA Transmission with an AF Relay, in IEEE Communications Letters, Vol. 21, No. 11, 2017, pp. 2428-2431.

[6] Z. Yang, Z. Ding, Y. Wu and P. Fan, Novel Relay Selection Strategies for Cooperative NOMA, in IEEE Transactions on Vehicular Technology, Vol. 66, No. 11, 2017, pp. 1011410123.

[7] G. O. Okeke, W. A. Krzymień, Y. Jing and J. Melzer, A Novel Low-Complexity Joint UserRelay Selection and Association for Multi-User Multi-Relay MIMO Uplink, in IEEE Wireless Communications Letters, Vol. 4, No. 3, 2015, pp. 309-312.

[8] F. Jameel, S. Wyne, S. J. Nawaz, Z. Chang and T. Ristaniemi, Outage Analysis of Relay-Aided Non-Orthogonal Multiple Access with Partial Relay Selection, in Proceedings of IEEE Global Communications Conference (Workshop), Abu Dhabi, United Arab Emirates, 2018, pp. 1-6.

[9] P. Xu, Z. Yang, Z. Ding and Z. Zhang, Optimal Relay Selection Schemes for Cooperative
NOMA, in IEEE Transactions on Vehicular Technology, Vol. 67, No. 8, 2018, pp. 78517855.

[10] S. Mondal, S. Dhar Roy, and S. Kundu, ClosedForm Outage Probability Expressions for Multihop Cognitive Radio Network with Best Path Selection Schemes in RF Energy Harvesting Environment, in Wireless Personal Communications, Vol. 103, No. 3 , 2018, pp2197-2212.

[11] N.S.Kim, Performance of User Relay Cooperative NOMA System with Partial and Imperfect CSI, in International Journal of Engineering and Technology, Vol. 7, No. 4, 2018, pp. 4462-4467.

[12] N.S.Kim, Performance of Max-Min User Relay Cooperative NOMA System with Imperfect CSI, in International Journal of Intelligent Engineering and Systems, Vol. 11, No. 6, 2018, pp. $148-155$.

[13] B. Zhong, Y. Li, J. Wang, Z. Zhang and K. Long, Partial Relay Selection with Fixed-Gain Relays and Outdated CSI in Underlay Cognitive Networks," $2013 \quad$ IEEE Wireless Communications and Networking Conference (WCNC), Shanghai, 2013, pp. 3670-3675.

[14] S. Arzykulov, T. A. Tsiftsis, G. Nauryzbayev and M. Abdallah, Outage Performance of Cooperative Underlay CR-NOMA With Imperfect CSI, in IEEE Communications Letters, 2019, Vol. 23, No. 1, pp. 176-179.

[15] S. Narmatha, R. Jeyanthi and N. Malmurugan, Amplify and Forward Relay Network Optimization with Imperfect CSI," in Proceedings of 2nd International Conference on Electronics and Communication Systems (ICECS), Coimbatore, 2015, pp. 469-473.

[16] M. Seyfi, S. Muhaidat and J. Liang, Amplifyand-Forward Selection Cooperation over Rayleigh Fading Channels with Imperfect CSI, in IEEE Transactions on Wireless Communications.Vol. 11, No. 1, 2011, pp. 199209.

[17] B. Zhong, Z. Zhang, X. Zhang, J. Wang and K. Long, Partial Relay Selection With Fixed-Gain Relays and Outdated CSI in Underlay Cognitive 
Networks, in IEEE Transactions on Vehicular Technology, Vol. 62, No. 9, 2013, pp. 46964701 .

[18] D. Do, M. Vaezi and T. Nguyen, Wireless Powered Cooperative Relaying Using NOMA with Imperfect CSI, in Proceedings of IEEE Globecom Workshops (GC Wkshps), Abu Dhabi, United Arab Emirates, 2018, pp. 1-6.

[19] T. Xing, N. Ma and P. Zhang, Two-Stage Power Allocation for Cooperative NOMA in D2D Communications with Imperfect CSI, in Proceedings of 11th International Conference on Wireless Communications and Signal Processing (WCSP), Xi'an, China, 2019, pp. 16.

[20] S. Abdel-Razeq, S. Zhou, R. Bansal and M. Zhao, Uplink NOMA Transmissions in a Cooperative Relay Network based on Statistical Channel State Information, in IET Communications, Vol. 13, No. 4, 2019 pp. 371378.

[21] A. Papadogiannis, Y. Sui and T. Svensson, "The Potential of a Hybrid Fixed/User Relay Architecture - A Performance Analysis,"in proceedings of IEEE Vehicular Technology Conference (VTC Fall), Quebec City, QC, pp. 15, 2012.

[22] D. Deng, L. Fan, X. Lei, W. Tan and D. Xie, "Joint User and Relay Selection for Cooperative NOMA Networks," in IEEE Access, vol. 5, pp. 20220-20227, 2017. 